\title{
Um Critério para Determinar a Regulagem da Tensão em Soldagem MIG/MAG por Curto-Circuito
}

\author{
(A Criterion to Determine Voltage Setting in Short-Circuit GMAW)
}

\author{
Gabriel Maradei Carneiro de Rezende ${ }^{1}$, Olga Liskévych ${ }^{1}$, Louriel Oliveira Vilarinho ${ }^{1}$, Américo Scotti ${ }^{1}$ \\ ${ }^{1}$ Universidade Federal de Uberlândia, Faculdade de Engenharia Mecânica, Grupo Laprosolda, Uberlândia-MG, Brasil \\ gabrielmcr@hotmail.com,liskevich@i.ua,vilarinho@mecanica.ufu.br,ascotti@mecanica.ufu.br
}

\begin{abstract}
Resumo
Este trabalho apresenta um estudo exploratório sobre um critério denominado "Critério Laprosolda para Estabilidade de Transferência em MIG/MAG com curto-circuito", o qual tem por finalidade uma análise quantitativa da regularidade da transferência metálica para regulagem de parâmetros para soldagem MIG/MAG por curto circuito convencional. Inicialmente é apresentado o embasamento para elaboração deste critério. A avaliação da confiabilidade do mesmo foi feita por meio de soldagens e comparações dos resultados com o rendimento de deposição e com as características geométricas do cordão de solda. Para isto, chapas de teste com 3,18 mm de espessura e abertura de raiz de $1 \mathrm{~mm}$ entre elas foram soldadas, dentro dos mesmos parâmetros de corrente e velocidade de alimentação, usando arame AWS ER70S-6 de 1,2 mm de diâmetro protegido pela mistura Argônio 85\% - Dióxido de Carbono $15 \%$. Para atender a relação proposta, varreu-se a regulagem de tensões de soldagem desde valores muito baixos até muito altos para o que se esperava para esta condição de soldagem. Os resultados confirmam a efetividade do "Índice de Regularidade" como meio de avaliar regulagens de soldagem MIG/MAG visando minimizar respingos e otimizar o acabamento do cordão.
\end{abstract}

Palavras chave: Soldagem, MIG/MAG, curto-circuito, transferência metálica, regulagem

Abstract: This paper presents an exploratory study on a criterion called "Criterion for Stability Laprosolda Transfer in MIG / MAG with short circuit", which aims at a quantitative analysis of the regularity of metal transfer for setting parameters for MIG / MAG conventional in short circuit. Initially it is stated the foundation for development of this criterion. The evaluation of that was made by welding and comparisons of results with the efficiency of deposition and the geometric characteristics of the weld bead. For this, the test plates with $3.18 \mathrm{~mm}$ thick and $1 \mathrm{~mm}$ gap between them was welded in the same range of current and feeding speed, using wire AWS ER70S-6 $1.2 \mathrm{~mm}$ of diameter protected by the mixture $85 \%$ Argon - carbon dioxide 15\%. To meet the proposed relationship, it was covered up the regulation of welding voltages from very low to very high for what was expected for this condition of welding. The results confirm the effectiveness of the "Index of regularity" as a means of assessing adjustments welding MIG / MAG to minimize spatter and optimize the coating on the weld bead.

Keywords: Welding, MIG / MAG, short circuit, metal transfer, adjustment

\section{Introdução}

Com finalidade de facilitar a realização das soldagens e, em conseqüência, a estimação da qualidade de solda, pesquisadores sempre procuram quantificar os fatores que influenciam na estabilidade do processo de soldagem. Segundo Ponomarev [1], a estabilidade do processo de soldagem MIG/MAG é avaliada por três fatores: estabilidade do arco, regularidade da transferência metálica e comportamento operacional do processo de soldagem, os quais se relacionam de forma muito próxima e complicada. Desta maneira, surgiu-se a necessidade de desenvolvimento de índices, que vão indicar estabilidade/instabilidade de ocorrência dos fenômenos indicados anteriormente. Até hoje, vários trabalhos foram feitos nessa área e, normalmente, eles são

(Recebido em 30/11/2010; Texto final em 11/04/2011).

Artigo originalmente publicado no COBEF 2010 baseados nas análises estatísticas das curvas de tensão do arco e da corrente elétrica utilizada no processo. Por exemplo, Arai et al. [2] mostram que há uma relação muito próxima entre o desvio padrão do tempo do arco aberto e a estabilidade do arco, baseado em experiência em soldagem MIG/MAG com transferência metálica por curto-circuito. Lucas [3] estudou a distribuição dos valores de desvios padrão dos picos de corrente de curto-circuito e seus períodos sob várias regulagens da tensão de soldagem, usando vários tipos de fontes de soldagem, e provou que há uma relação entre estes desvios padrão e a estabilidade do arco. Mita et al. [4] obtiveram um desvio padrão dos fatores das curvas de tensão do arco e da corrente de soldagem (tempo de arco aberto, tempo de curto-circuito, corrente media e corrente media de curto-circuito, etc.). Esses autores propuseram alguns modelos de regressão, os quais foram compostos por elementos tirados dos oscilogramas (por exemplo, tempos de arco aberto, tempos de curto-circuito, picos de corrente de curto-circuito, etc.) e seus desvios padrão. Eles também fizeram um modelo composto baseado na avaliação de soldadores experientes e 
consideraram esse modelo como um índice de estabilidade do arco. Mostraram ainda que quando o índice era alto, o arco ficava instável, e, quando o índice era baixo, o arco podia ser estimado como estável. Outro trabalho, de Kang e Rhee [5], mostra um modelo otimizado de estimação para a quantidade de respingos, quando se considera a extinção do arco operando em transferência metálica por curto-circuito, usando análise de regressão múltipla.

$\mathrm{Na}$ verdade, a maioria dos trabalhos utiliza a regularidade dos elementos dos oscilogramas ou a relação entre sentimentos do soldador e alguns modelos para estimar o comportamento operacional do processo de soldagem MIG/MAG, mas nenhum deles considera estabilidade do arco e regularidade da transferência metálica em conjunto.

O Grupo Laprosolda criou um critério para quantificar a estabilidade de transferência por curto-circuito no processo de soldagem MIG/MAG [6]. Este critério se baseia na premissa de que a estabilidade da transferência por curto-circuito está ligada com a constância dos tempos em curto-circuito e com arco aberto, assim como com o fato de que cada gota ao se destacar tenha um volume apropriado para haver a ação da tensão superficial para se obter a transferência. Assim, esse critério tem de satisfazer dois parâmetros, a saber:

1) Índice Vilarinho de Regularidade da Transferência por Curto-circuito, cujo símbolo é IVcc. Este índice é determinado por intermédio dos coeficientes de variação dos tempos de curtocircuito (tcc) e de arco aberto (tab), como mostra a Eq. 1.

$\mathrm{IV}_{\mathrm{cc}}=\frac{\sigma_{\mathrm{tcc}}}{\mathrm{t}_{\mathrm{cc}}}+\frac{\sigma_{\mathrm{tab}}}{\mathrm{t}_{\mathrm{ab}}}$

Onde $\sigma_{\text {tcc }}=$ desvio padrão da média do tempo de curtocircuito; $\sigma_{\text {tab }}=$ desvio padrão da média do tempo de arco aberto, $\mathrm{t}_{\mathrm{cc}}=$ média do tempo de curto-circuito; $\mathrm{t}_{\mathrm{ab}}=$ média do tempo de arco aberto.

2) Faixa admissível de tamanho de gota em transferência por curto-circuito, cujo símbolo é $\Delta \mathrm{Fcc}$. Essa faixa define os volumes da gota em transferência a cada curto-circuito, de tal forma a permitir um "sugamento" suave da mesma pela poça (efeito da tensão superficial como principal mecanismo). Pode-se supor que uma gota não deva crescer muito antes de se destacar. Por outro lado, precisa ter um volume mínimo. Naturalmente esta faixa vai ser função do material do arame e do gás de proteção. Porém, medir tamanho médio de gota é uma tarefa laboriosa e requer equipamentos especiais. Mas pode-se estimar esta faixa de volume adequado pelo cálculo das freqüências máximas (Fccmax) e mínimas (Fccmin) esperadas, conforme Eq. (2).

$\mathrm{F}_{\mathrm{cc}}=\frac{\mathrm{V}_{\mathrm{alim}} \cdot(\mathrm{d})^{2}}{(\mathrm{k} \cdot \mathrm{d})^{3}}$

Onde $\mathrm{d}$ = diâmetro do arame-eletrodo em milímetros; $\mathrm{V}_{\text {alim }}=$ velocidade de alimentação de arame em milímetros por segundo; $\mathrm{k}=$ fator de multiplicação para estimar o diâmetro da gota em função do diâmetro do arame.
Para freqüência mínima, usa-se como k o valor que se refere a gotas de tamanho máximo, e para frequiência máxima, usase como $\mathrm{k}$ o valor que se refere a gotas de tamanho mínimo. Como mencionado, o valor de $\mathrm{k}$ depende do material do arame e do gás de proteção e ainda precisa ser mais bem determinado com filmagens de alta velocidade para diferentes combinações arame/gás de proteção.

Desta forma, neste trabalho se pretende avaliar a aplicação desse critério como forma de ser um meio quantitativo simples, porém seguro, de se determinar a faixa de regulagem de tensão que garanta uma maior estabilidade de transferência metálica em soldagem MIG/MAG por curto-circuito.

\section{Metodologia}

\subsection{Procedimento Experimental}

Para avaliação prática do critério proposto, foram realizados testes de soldagens por curto-circuito no processo MIG/ MAG, utilizando-se o arame-eletrodo AWS ER70S-6, com diâmetro de $1,2 \mathrm{~mm}$ e como gás de proteção uma mistura de Argônio com 15\% de Dióxido de Carbono. A composição do gás, devido a importância para este estudo, foi aferida num analisador comercial, apresentando o valor de $14,8 \% \mathrm{em}$ média. Para uma dada corrente, a tensão de regulagem na fonte foi sistematicamente alterada de valor $(16,17,18,19,20,22$ e 24 Volts), de modo a se obter desde comprimentos de arcos muito curtos a muito longos. Para cada uma das soldagens com essas tensões de regulagem, foram medidos e/ou calculados o rendimento de deposição, o Índice de Regularidade, a geometria do cordão e a frequência de curtos-circuitos.

A fonte utilizada foi um equipamento de soldagem de uso comercial (Lincoln Powerwave 450 STT), trabalhando no modo MIG convencional (tensão constante) e com a regulagem da indutância na posição média. Como a indutância no arco de soldagem depende de outros fatores, mas principalmente do equipamento e sua regulagem do fator indutivo, torna-se necessário neste texto especificar o equipamento, como base de referência. Por outro lado, determinar e especificar o valor da taxa de variação de corrente durante os curtos-circuitos pareceu aos autores desnecessário para o fico do trabalho, frente a influência da tensão e gás de proteção sobre a correlação entre a indutância e a regularidade da transferência metálica na soldagem MIG/ MAG por Curto-Circuito, como apontado por Souza et al. [7]. A corrente de trabalho escolhida foi no valor de $125 \mathrm{~A}$, coerente com a espessura da chapa de teste (em seguida especificada) e com o tipo da transferência metálica requerida (curto-circuito). Procurou-se um valor próximo ao limite inferior da faixa útil de corrente para este modo de transferência (para o dado gás de proteção e arame-eletrodo), para evidenciar mais ainda a regulagens de pouca estabilidade. Esse valor foi regulado com uma velocidade de alimentação fixa de $2,46 \mathrm{~m} / \mathrm{min}$. A distância bico de contato-peça (DBCP) foi arbitrariamente escolhida como $12 \mathrm{~mm}$.

Como placas de teste, foram utilizadas barras chatas de aço carbono, de 3,18 $\mathrm{mm}$ de espessura e $19 \mathrm{~mm}$ de largura, com uma abertura de raiz de $1 \mathrm{~mm}$ entre elas (com a chapa faceada) 
para configurar uma junta com chanfro em I (condição mais semelhante à prática), conforme a Fig. 1. Para assegurar a abertura de raiz, foram feitos três pontos equidistantes de solda entre as barras, a partir do começo delas. Na realização das soldagens, estas placas foram acopladas em um suporte (Fig. 2), o qual promove uma fixação adequada da placa de teste para que esta não sofra distorção durante soldagem. Este suporte está sobre uma mesa de soldagem sobre a qual se movimenta um braço robótico, onde a tocha foi anexada como mostra a Fig. 3.

\subsection{Métodos de Análise}

Para encontrar o rendimento de deposição, utilizou-se o valor da massa da chapa antes e depois da realização do cordão de solda. Da diferença das massas (levando-se em conta o cuidado na retirada de respingos que ficam na chapa), tem-se como resultado a massa real de material depositada na mesma, que é a própria massa do cordão de solda. Conhecendo-se a densidade, o diâmetro e o comprimento do arame utilizado (este último calculado a partir do tempo de arco aberto e da

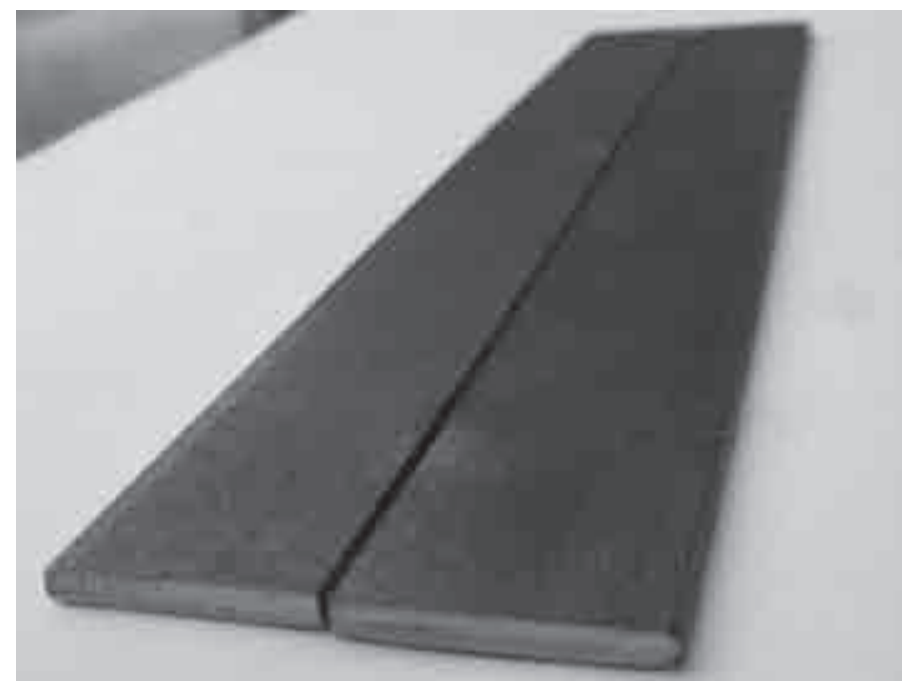

Figura 1: Ilustração da placa de testes velocidade de alimentação), é possível obter a massa de arame que foi alimentado. Divide-se a massa do cordão pela massa de arame alimentado para, assim, obter o rendimento (comumente expresso em porcentagem).

Para encontrar o Índice Vilarinho de Regularidade, utilizase um programa dedicado desenvolvido no ambiente MATLAB, o qual calcula numericamente os valores e respectivos desvios padrões da Eq. 1 (esses dados são mostrados na Tab. 2). Vale ressaltar que cada tempo foi calculado por uma média aritmética dos valores tomados em dois trechos da chapa, para que o ponto de solda central de fixação não influenciasse nos resultados. Este programa também calcula e fornece a frequência de curtoscircuitos (Fcc) durante o processo. O cálculo dos valores limites de frequência mínima e máxima foram feitos usando-se a Eq. 2, tomando como base, de forma arbitrária (observando a transferência), diâmetros de gota aceitáveis como de 1,15 a 1,30 vezes o diâmetro do arame, respectivamente.

A geometria do cordão foi analisada por meio de macrografias de duas seções transversais obtidas por corte em posições aleatórias ao longo do cordão de solda. Essas seções

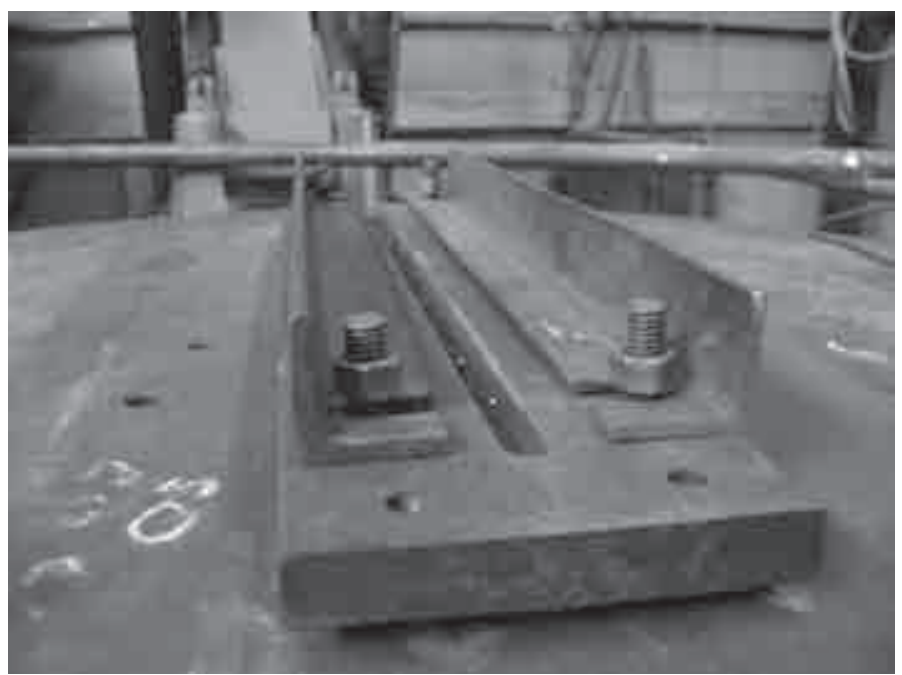

Figura 2: Suporte para placa de testes

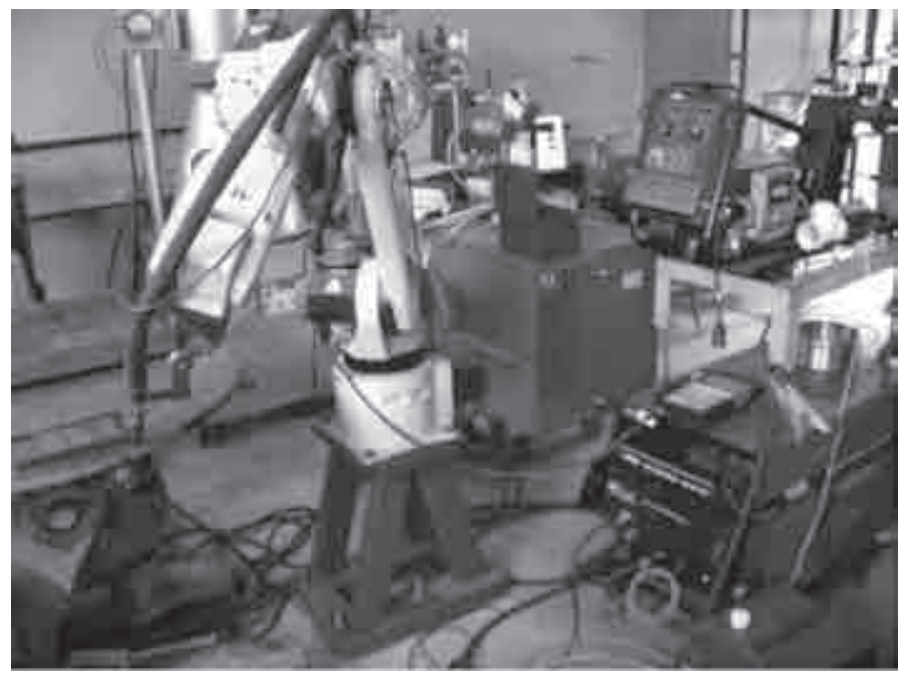

Figura 3: Fonte, robô e mesa de soldagem 
são devidamente preparadas para metalografia (lixadas, polidas e atacadas com Nital 10\%) para evidenciar a área fundida e área termicamente afetada. Sobre as macrografias de cada cordão calculou-se o valor médio da área fundida e da penetração. Para a análise das características geométricas do cordão de solda mediu-se também a altura do reforço e a largura do cordão com um paquímetro digital (0,01 mm de precisão).

\section{Resultados e Discussões}

Durante a varredura de tensões na regulagem da fonte de soldagem, fixou-se uma velocidade de alimentação de 2,46 m/ min (valor indicado no painel do equipamento, cuja calibração foi aferida previamente) buscando uma corrente próxima de 125 A para a dada DBCP selecionada. A Tabela 1 apresenta os valores resultantes deste procedimento, enquanto a Tabela 2 apresenta os dados computados pelo programa acima mencionado, utilizados nos cálculos do índice IVcc.

Tabela 1: Tensões e correntes monitoradas em função da tensão e velocidade de alimentação reguladas no equipamento

\begin{tabular}{|c|c|c|c|c|}
\hline $\begin{array}{c}\text { Tensão } \\
\text { regulada } \\
{[\mathrm{V}]}\end{array}$ & $\begin{array}{c}\text { Tensão } \\
\text { Média } \\
{[\mathrm{V}]}\end{array}$ & $\begin{array}{c}\text { Tensão } \\
\text { RMS } \\
{[\mathrm{V}]}\end{array}$ & $\begin{array}{c}\text { Corrente } \\
\text { Média } \\
{[\mathrm{A}]}\end{array}$ & $\begin{array}{c}\text { Corrente } \\
\text { RMS } \\
{[\mathrm{A}]}\end{array}$ \\
\hline 16 & 16,3 & 16,9 & 126,8 & 143,8 \\
\hline 17 & 17,2 & 17,8 & 128,1 & 143,1 \\
\hline 18 & 18,5 & 19,2 & 118,8 & 125,3 \\
\hline 19 & 19,4 & 19,8 & 127,3 & 138,1 \\
\hline 20 & 20,3 & 20,9 & 122,2 & 131,6 \\
\hline 22 & 22,1 & 22,3 & 118,9 & 125,5 \\
\hline 24 & 23,8 & 24,0 & 123,3 & 127,1 \\
\hline
\end{tabular}

Obs: RMS se refere ao valor eficaz (root mean square)

Tabela 2: Valores médios característicos dos curtoscircuitos função da tensão de regulagem.

\begin{tabular}{|c|c|c|c|c|}
\hline Tensão [V] & $\mathrm{t}_{\mathrm{ab}}[\mathrm{s}]$ & $\mathrm{t}_{\mathrm{cc}}[\mathrm{s}]$ & $\sigma_{\mathrm{tab}}[\mathrm{s}]$ & $\sigma_{\mathrm{tcc}}[\mathrm{s}]$ \\
\hline 16 & 0,0168 & 0,0024 & 0,0071 & 0,0006 \\
\hline 17 & 0,0205 & 0,0025 & 0,0087 & 0,0004 \\
\hline 18 & 0,0329 & 0,0034 & 0,0090 & 0,0005 \\
\hline 19 & 0,0352 & 0,0026 & 0,0116 & 0,0005 \\
\hline 20 & 0,0507 & 0,0040 & 0,0183 & 0,0007 \\
\hline 22 & 0,1770 & 0,0049 & 0,1123 & 0,0011 \\
\hline 24 & 0,2774 & 0,0041 & 0,1322 & 0,0008 \\
\hline
\end{tabular}

Onde: $t_{a b}$ e $t_{c c}$ se referem aos tempos de arco aberto e curto circuito, respectivamente, com seus respectivos desvios padrão $\left(\sigma_{t a b} e \sigma_{t c c}\right)$.

A Tabela 3 e Figuras 4 e 5 foram desenvolvidas para facilitar a análise dos resultados. Pela Tab. 3, pode-se perceber que os rendimentos de deposição (Rend.) foram altos, mas que há uma tendência de valores máximos no centro da faixa (ilustrado pela Fig. 4). Também se observa que a frequência medida das transferências $(\mathrm{Fcc})$ é decrescente à medida que a tensão regulada aumenta, mas que apenas na faixa mais central o valor medido se enquadra na faixa entre as frequências mínima (Fcc min) e máxima (Fcc max). Finalmente, o índice para medir a regularidade da transferência (IVcc) parece, também, mostrar uma tendência de mínimo (quanto menor, melhor) no centro da faixa, como visto na Fig. 4. Comparando-se essas figuras, é possível observar uma correlação no comportamento entre rendimento de deposição e IVcc; há um ponto de máximo para o rendimento coincidente com o de mínimo para o Índice de Regularidade, no que seria a melhor tensão para a soldagem (18 $\mathrm{V}$ no caso). Nas tensões mais afastadas deste ponto, é clara a percepção de um comportamento da transferência com menor rendimento e pior regularidade, o que confirma em parte o postulado proposto.

Ainda pela Tab. 3, é possível perceber, por intermédio da baixa frequência de curtos-circuitos, que para tensões maiores ou iguais a $22 \mathrm{~V}$ a deposição de material começa a perder sua característica de transferência por curto-circuito, passando para uma faixa de transição para transferência globular. É importante apontar que a transferência tendendo para globular $(24 \mathrm{~V})$ pode apresentar alta regularidade (medida pelo baixo valor de IVcc nesta tensão), razão pela qual se está usando um critério de corte pela frequência, como proposto neste trabalho. Por outro lado, com a regulagem de tensões mais baixas a frequência é alta, mas a regularidade medida pelo IVcc também é alta (menor regularidade).

Tabela 3: Resultados gerais (em função da tensão de regulagem) para os rendimentos, freqüências de curtos circuitos e Índice de Regularidade.

\begin{tabular}{|c|c|c|c|c|c|}
\hline $\begin{array}{c}\text { Tensão } \\
(\mathrm{V})\end{array}$ & Rend. & $\begin{array}{c}\text { Freq. } \\
\text { Mín. [Hz] }\end{array}$ & $\begin{array}{c}\text { Freq. } \\
{[\mathrm{Hz}]}\end{array}$ & $\begin{array}{c}\text { Freq. } \\
\text { Máx. [Hz] }\end{array}$ & IVcc \\
\hline 16 & $95,0 \%$ & 23,7 & 43,2 & 34,3 & 0,7 \\
\hline 17 & $95,8 \%$ & 23,7 & 37,0 & 34,3 & 0,6 \\
\hline 18 & $96,6 \%$ & 23,7 & 27,8 & 34,3 & 0,4 \\
\hline 19 & $95,9 \%$ & 23,7 & 23,6 & 34,3 & 0,5 \\
\hline 20 & $95,3 \%$ & 23,7 & 18,0 & 34,3 & 0,5 \\
\hline 22 & $94,9 \%$ & 23,7 & 4,5 & 34,3 & 0,8 \\
\hline 24 & $94,1 \%$ & 23,7 & 3,0 & 34,3 & 0,7 \\
\hline
\end{tabular}

Uma análise mais abrangente pode ser feita observandose a Fig. 6, pela qual é possível perceber a relação entre a regularidade e a frequência de transferência e a tensão de regulagem para soldagem. Para facilitar o entendimento, nesta figura foram impostas duas faixas de incerteza (transição), sendo que a inferior determina a faixa mínima de frequência na qual a transferência ainda ocorre com estabilidade, enquanto que a faixa superior determina a frequência máxima. Para a faixa de frequência mínima, impôs-se como base uma gota fundida com diâmetro entre 1,30 e 1,35 vezes maior que o diâmetro do arame, e não somente 1,30 vezes o diâmetro do arame. Para a faixa de frequência máxima impôs-se, de forma análoga, uma 


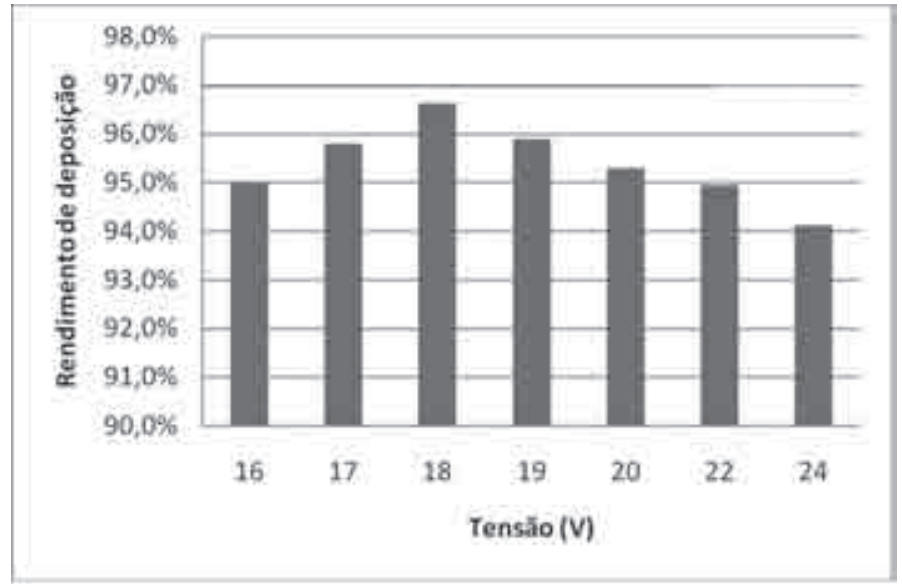

Figura 4: Resultados do rendimento em função da tensão de regulagem

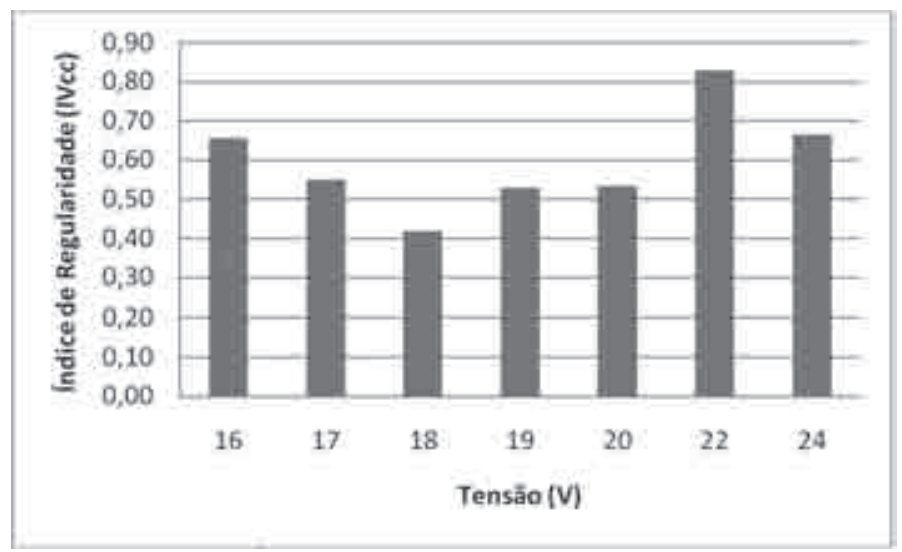

Figura 5: Resultados do Índice de Regularidade em função da tensão de regulagem

gota fundida com o diâmetro entre 1,10 e 1,15 vezes maior que o diâmetro do arame. Estes valores de alargamento do valor mínimo e máximo, passando a ser caracterizado por uma faixa de incerteza, foram também atribuídos de forma arbitrária pela experiência e expectativas do grupo de soldagem Laprosolda.

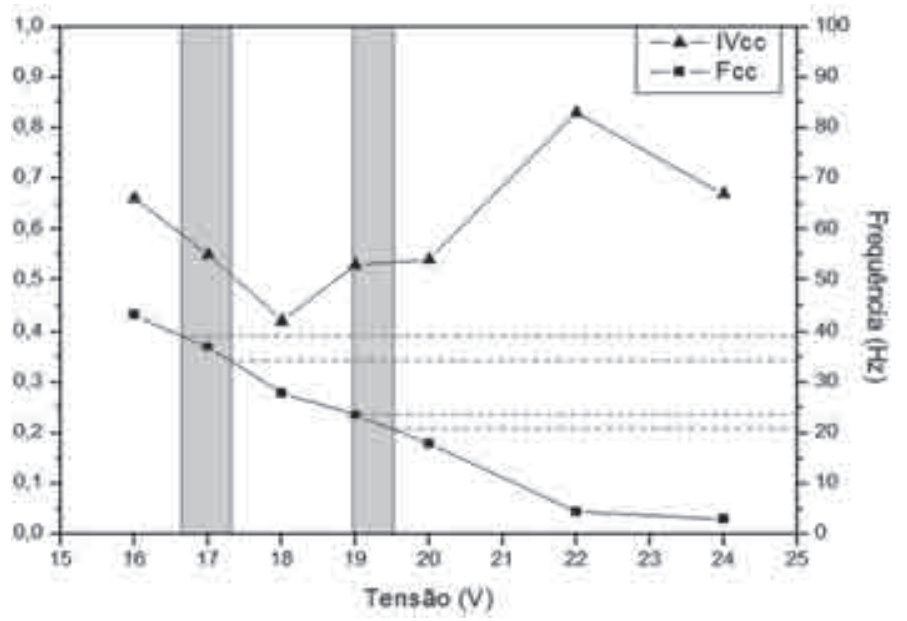

Figura 6: Faixas de transição dos limites superiores e inferiores da tensão de soldagem em que os IVcc são aceitáveis, em função das faixas de freqüência máxima e mínima adequadas para haver a transferência com estabilidade

Tomou-se em conta principalmente a existência de alta estabilidade de transferência (baixo IVcc) próxima aos valores inicialmente determinados como limites. Então, de acordo com esses critérios, as tensões de 17 a $18 \mathrm{~V}$ representariam a melhor condição de soldagem, valendo lembrar que as tensões de 16 até aproximadamente 17 e de aproximadamente 18 até $19 \mathrm{~V}$ se encontram na região de incerteza.

A Tabela (4) apresenta a caracterização geométrica dos cordões produzidos. De acordo com as macrografias mostradas na Fig. 7, observa-se também uma relação entre a tensão e as características geométricas do cordão de solda. Em transferências mais regulares (neste caso, o de máximo em $18 \mathrm{~V}$ ), tem-se uma maior área fundida, uma maior penetração e um cordão com convexidade adequada. Já em tensões mais afastadas da faixa ótima, tem-se um maior reforço e menor área fundida (naturalmente, a geometria é afetada pelo comprimento do arco e tempos de arco aberto e em curto-circuito, fatores que o critério de estabilidade conseguiu deixar intrínsecos).

Tabela 4: Valores geométricos do cordão de solda.

\begin{tabular}{|c|c|c|c|c|}
\hline Tensão [V] & Área fund. $\left[\mathrm{mm}^{2}\right]$ & Penetração [mm] & Reforço [mm] & Largura [mm] \\
\hline 16 & $6,31 \pm 1,22$ & $2,60 \pm 0,56$ & $1,80 \pm 0,14$ & $6,03 \pm 0,22$ \\
\hline 17 & $6,59 \pm 0,54$ & $2,28 \pm 0,15$ & $2,03 \pm 0,07$ & $6,74 \pm 0,30$ \\
\hline 18 & $8,61 \pm 0,63$ & $3,10 \pm 0,10$ & $1,32 \pm 0,09$ & $6,28 \pm 0,24$ \\
\hline 19 & $7,44 \pm 0,40$ & $1,98 \pm 0,15$ & $1,82 \pm 0,11$ & $7,40 \pm 0,31$ \\
\hline 20 & $6,34 \pm 0,71$ & $2,07 \pm 0,28$ & $1,82 \pm 0,12$ & $6,77 \pm 0,36$ \\
\hline
\end{tabular}



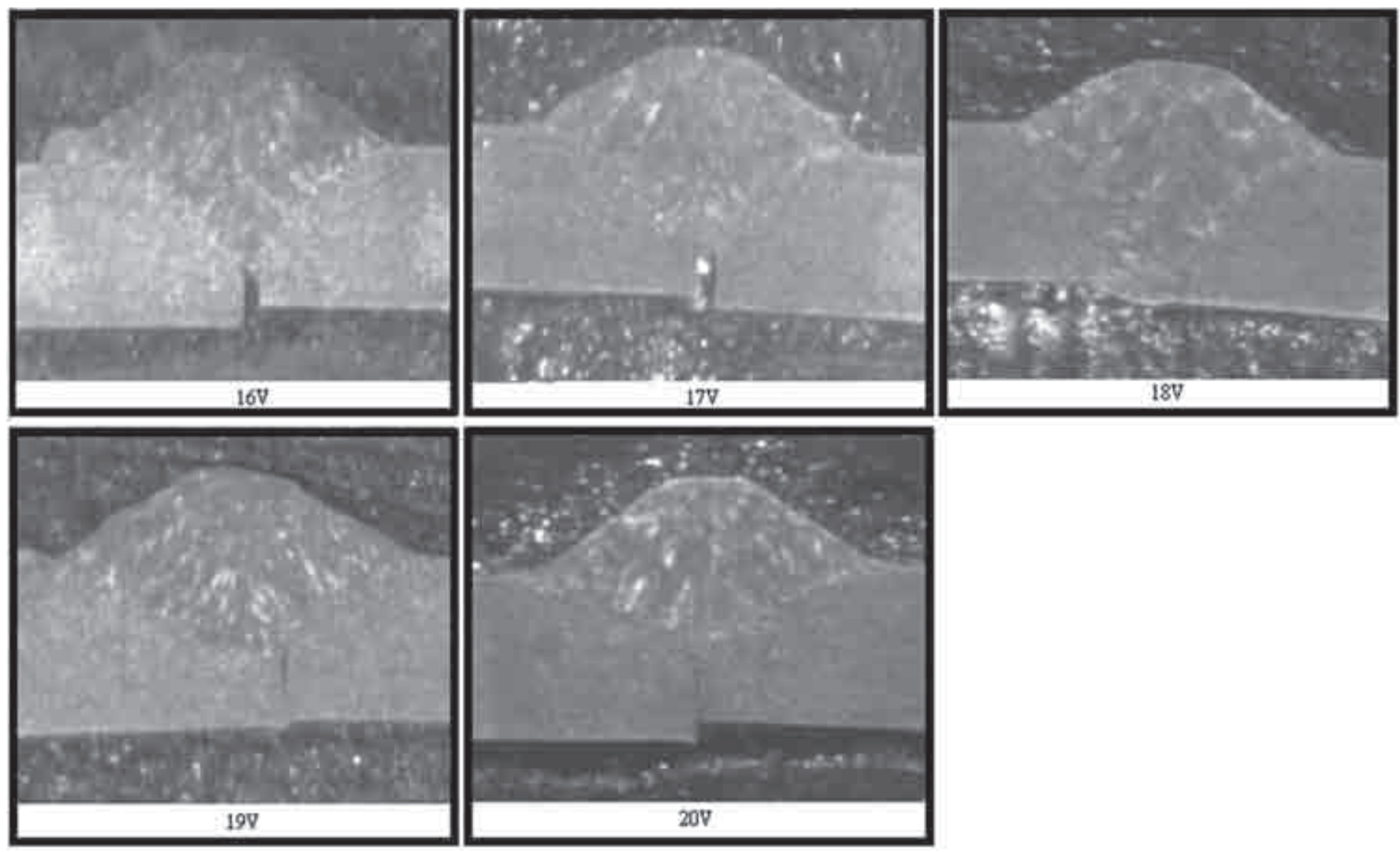

Figura 7: Seções transversais dos corpos de prova, para cada tensão de regulagem (em destaque a área fundida e a penetração da solda)

\section{Conclusão}

Os resultados confirmam o postulado de que a estabilidade de transferência em soldagem MG/MAG com curto-circuito pode ser medida por meio do índice Vilarinho de regularidade (IVcc), desde que aplicado uma restrição baseada em um corte pelo afastamento do tamanho adequado das gotas. Assim, a faixa de regulagem de tensão que garanta uma maior estabilidade de transferência metálica em soldagem MIG/MAG por curtocircuito pode ser quantitativamente determinada e monitorada em uma linha de produção.

\section{Agradecimentos}

Os autores agradecem Faculdade de Engenharia Mecânica da UFU, através de seu Centro para Pesquisa e Desenvolvimento de Processos de Soldagem (Laprosolda), pela oportunidade de utilização dos equipamentos e do suporte técnico disponibilizado, e as agências FAPEMIG (proc. TEC 604/05), pela concessão de recursos financeiros, e CNPq, pela concessão de bolsas de estudo e pesquisa para os autores.

\section{Referencias Bibliográficas}

[1] PONOMAREV, V., 1997, "Arc process statistical analysis", JOM-Institute, DTU-Helsingor, Denmark, pp. 59-100.
[2] ARAI, T.; KOBAYASHI, M.; YAMADA, T.; ROKUJIYO, M.; HIRAKOSO, K.; KANEKO, T., 1983, "The investigation of arc phenomena by means of computer". Quarterly Journal of the Japan Welding Society 1(3), pp. 15-20.

[3] LUKAS, W., 1987, "Microcomputer system, software and expert system for welding engineering", Welding Journal 66(4), pp. 19-30.

[4] MITA, T.; SAKABE, A.; YOKOO, T., 1988., Quantitative estimates of arc stability for $\mathrm{CO}_{2}$ gas shielded arc welding., Welding International 2(2), pp. 152-159.

[5] KANG, M. J.; RHEE, S., 2001, "The Statistical Models for Estimating the Amount of Spatter in the Short Circuit Transfer Mode of GMAW", Welding Journal, January, pp. 1s-8s.

[6] SOUZA, D. et al., Relatório Interno Laprosolda/UFU 13/2009 - Manual de Utilização do Programa de Análise de CurtoCircuito para Determinação da Estabilidade de Transferência no Processo de Soldagem MIG/MAG, 9p.

[7] SOUZA, D., ROSSI, M.L., KEOCHEGUERIANS, F., NASCIMENTO, V.C., VILARINHO, V.L., SCOTTI, A. Influência da Tensão de Soldagem e do Gás de Proteção sobre a Correlação entre Indutância e Regularidade da Transferência Metálica na Soldagem MIG/MAG por Curto-Circuito, Soldagem \& Inspeção, Vol. 16, No. 1, Jan/Mar 2011, pp. 22-32 (ISSN 0104-9224 printed/ISSN 1980-6973 on-line. 